\title{
Role of Diet and Diet Interventions in Diabetic Patients: Physiological and Metabolic Changes and Reduction in Morbidity and Mortality
}

\author{
Rosalba Giacco • Claudia Vetrani - Ettore Griffo • \\ Angela A. Rivellese
}

Published online: 13 October 2013

(C) Springer Science+Business Media New York 2013

\begin{abstract}
Evidence of the impact of diet and dietary components on cardiovascular risk factors and morbidity/mortality in diabetic patients over the last 3 years is reviewed. The review comprises a section focusing on the effects of diet on body weight reduction and other cardiovascular risk factors, and a section in which recent evidence on hard endpoints are discussed. Overall, the data accumulated in the period considered seem to confirm the recommendations for diabetes management. More intervention studies focusing on hard endpoints in diabetic subjects are needed to prove in a definitive way the role of diet on the reduction of cardiovascular morbidity and mortality, especially that concerning weight reduction.
\end{abstract}

Keywords Diabetes · Dietary pattern · Risk factors · Cardiovascular events · Cardiovascular mortality

\footnotetext{
C. Vetrani $\cdot$ E. Griffo • A. A. Rivellese $(\bowtie)$

Department of Clinical Medicine and Surgery, "Federico II"

University of Naples, Via S. Pansini 5, 80100 Naples, Italy

e-mail: rivelles@unina.it

C. Vetrani

e-mail: c.vetrani@libero.it

E. Griffo

e-mail: ettoregri@gmail.com

R. Giacco

Institute of Food Science, CNR- National Research Council,

Via Roma 64, 83100 Avellino, Italy

e-mail: rgiacco@isa.cnr.it
}

\section{Introduction}

Diet is recognized as one of the cornerstones in the treatment of diabetes, having as primary goals not only the improvement in blood glucose control and other metabolic cardiovascular (CV) risk factors $[1,2]$, but also the reduction of cardiovascular disease (CVD), which accounts for about $70 \%$ of total mortality in these patients [3].

Moreover, since diet and its components may act on the reduction of $\mathrm{CV}$ risk through different mechanisms, having pleiotropic effects, a healthy diet may be particularly important in patients characterized by the aggregation of multiple risk factors (overweight/obesity, poor glycemic control, dyslipidemia and high blood pressure). Nevertheless, little is known about this task in diabetic patients, at least for what concerns intervention studies with hard endpoints, such as incidence of CV morbidity and mortality.

Hence, this review will mainly take into account the papers published in the last 3 years that have evaluated the effects and possible mechanisms of action of the diet on metabolic parameters, CVD risk factors, and CV morbidity and mortality in diabetic patients.

Since some studies have investigated the role of diet in primary prevention of diabetes, a brief summary of the evidence on this aspect is included.

\section{Diet and Type 2 Diabetes Prevention}

As reported above, almost $70 \%$ of total mortality in patients with type 2 diabetes (T2D) is due to CVD. Since T2D is increasing throughout the world, a reduction in CVD could 
be achieved by preventing the development of this disease, which is strongly linked to westernized dietary patterns, physical inactivity and rising rates of obesity.

In the last three decades, several studies have tried to clarify the relation existing between food groups or dietary patterns and the risk of T2D, as well the effects of lifestyle modification and dietary changes on the risk to develop T2D.

More recently, two observational studies have provided further information on the role of food consumption or Mediterranean diet on T2D risk. The first, the EPIC-Potsdam study, has confirmed in line with previous studies that higher intake of whole-grain bread, fruits, raw vegetables and coffee is inversely associated with T2D risk in a large cohort of healthy subjects, during an average follow-up of 8 years [4]. Conversely, high intake of red meat, butter, sauces and low-fat dairy is associated with an increase of T2D risk.

The InterAct study has confirmed that an higher adherence to a Mediterranean dietary pattern is associated with a significant reduction $(-12 \%)$ in the risk of developing T2D compared with individuals with lower adherence to Mediterranean diet in a large cohort of healthy subjects from Mediterranean and non-Mediterranean countries [5].

The role of dietary patterns, in particular of the Mediterranean diet, in the reduction of T2D risk has been clearly reinforced by results of the PREDIMED study, investigating the effects of two Mediterranean diets (MedD) supplemented with extra-virgin olive oil or mixed nuts versus a low-fat diet on incidence of diabetes in individuals of middle age having at least three CV risk factors [6]. After a median follow-up of 4 years, a multivariable adjusted hazard ratio has been almost $50 \%$ lower in the participants assigned to the two MedD compared to the control diet. In addition, in all arms of the study, increased adherence to MedD has been inversely associated with diabetes. We underline that in this study, the reduction of T2D risk has been observed in the absence of significant changes in body weight or physical activity, suggesting that the mechanisms involved in diabetes risk reduction are independent of body weight loss and could be linked to other mechanisms, such as improvement in $\beta$-cell function, reduction of oxidative stress and inflammation.

In addition to the effect of diet composition, new data from the Diabetes Prevention Study (DPS) have further outlined the importance of overall lifestyle modifications on the reduction of T2D; in fact, the benefits of moderate weight reduction, together with an increase of physical activity, are preserved in the long-term, even many years after the conclusion of the intervention [7].

Although lifestyle interventions are not easily applicable in real-life settings, the European Diabetes Prevention Study (EDIPS) has recently shown that the DPS protocol can be applied in other European countries with success, reducing by $57 \%$ the cumulative T2D incidence during a mean follow-up of 3.1 years [8].

\section{Diet in Diabetic Patients}

This section will address evaluating the effects of different diets on body weight and other $\mathrm{CV}$ risk factors.

\section{Body Weight}

Overweight and obesity are the most important modifiable risk factors for T2D and CVD. As a matter of fact, it is well known that abdominal adiposity induces insulin resistance and, consequently, an increased steady requirement for insulin secretion. This condition represents a stressful state that can lead, over the time, to $\beta$-cell function decline with deterioration of blood glucose control. Moreover, excessive body weight is associated with an increase in many of the most important $\mathrm{CV}$ risk factors.

Therefore, in overweight or obese individuals with T2D, evidence-based recommendations suggest that weight loss is the first step of T2D management, because the improvement of insulin resistance and of $\beta$-cell function may be able to improve glucose metabolism, induce partial or total remission of diabetes and reduce the other $\mathrm{CV}$ risk factors linked to overweight/obesity.

In the last 3 years, some studies have clearly shown the benefits of intensive dietary interventions and lifestyle changes on body weight and metabolic parameters.

Intensive individualized dietary intervention, based on the nutritional recommendations of the European Association for the Study of Diabetes, is able to improve blood glucose control, and reduce body weight and waist circumference compared to usual care in diabetic patients not adequately controlled despite optimized hypoglycemic treatment [9].

Regarding lifestyle changes, the long-term results of the Action for Health in Diabetes trial (Look AHEAD) have been published. The Look AHEAD trial is the first study that has investigated the effects of a moderate body weight reduction, obtained by an intensive lifestyle intervention that combined a moderate energy restriction of diet with a daily increase of physical activity, on several $\mathrm{CV}$ risk factors and the incidence of CV events and mortality in a large cohort of overweight and obese individuals with T2D. In relation to CV risk factors, results of the study have shown that the intensive lifestyle intervention, compared with an usual education program, represents a good strategy to reduce body weight, and significantly improve blood pressure and metabolic profile in the long-term (4 years of follow-up) [10••]. In addition, in a small number of patients, the intervention is even able to induce a partial or total remission of type 2 diabetes [11]. Noteworthy is the sustained effect of lifestyle intervention on HDLcholesterol (HDL-col). In contrast to several other risk factors, the effect for HDL-col is greater at 4 years than at 1 year; at each year, HDL-col in the lifestyle group is approximately 8$9 \%$ higher than baseline levels, whereas in the control group, 
it is 3-6\% above baseline. Interestingly, although severely obese participants have not reached their ideal body weight, a significant reduction of blood pressure, glucose, HbAlc and triglycerides has been achieved, confirming the benefits of moderate weight loss (7-10\% of initial body weight) in the management of T2D metabolic abnormalities. However, the improvement in $\mathrm{CV}$ risk factors reported in this study has been achieved with great investment in terms of economic and professional resources; hence, this approach is not easily applicable in clinical practice, and more feasible strategies should be identified.

An easier approach based on additional time for dietary support (6.5 hours/years more than usual clinical practice) has been applied in the Early ACTID trial [12••] within the context of UK National Health Service. The results have confirmed the effectiveness of dietary intervention to reduce body weight and medication need, and to improve glucose homeostasis in 1 year's time. With respect to the increase of physical activity, no additional benefits have been observed for risk factor improvement or on the magnitude of body weight reduction. It can be hypothesized that this result is due to the lack of real increase in physical activity, since only general advice was given to participants, rather than a controlled physical exercise program.

Considering the importance of weight reduction and the challenge in achieving and maintaining stable weight loss, much attention has been always placed on the most effective dietary composition to decrease body weight and improve other CV risk factors in people with T2D. In this respect, a recent meta-analysis [13•] has tried to give answers to this question. On the basis of 20 clinical trials, the results of this metaanalyses seem to suggest that: a) different types of diet (lowcarbohydrate, low-glycemic index, Mediterranean and highprotein diets) are all effective in blood glucose control; b) lowcarbohydrate and Mediterranean diets are more effective in reducing body weight. However, as pointed out in the editorial done on this meta-analyses [14], the results should be considered with caution because the studies analyzed are few-especially for some outcomes (i.e. body weight) - quite heterogeneous, and the control diet is often not very well characterized.

Hence, this new evidence reinforces the concept that energy intake reduction seems to be more important than the qualitative composition of the diet, at least for weight loss, as we have recently reviewed [15].

In relation to energy restriction, the potential mechanisms triggered by it, independent from body weight loss, have been investigated by two recent trials, focusing on the effects of a verylow calorie diet (VLCD) in patients with T2D [16, 17]. Findings of these two studies demonstrate that short-term (1 week) caloric restriction per se can improve glucose control, $\beta$-cell function and insulin sensitivity, independently from weight-loss.

In particular, Lim and colleagues [16] have demonstrated a clear "multi-step" mechanism behind the observed effects, primarily involving changes in hepatic insulin sensitivity, followed by a slower change in the $\beta$-cell function. Moreover, the normalization of both $\beta$-cell function and hepatic insulin sensitivity in T2D is associated with decreased pancreatic and liver triglycerides content, which has been linked to T2D etiology.

\section{Other Cardiovascular Risk Factors}

Several trials have been carried out to assess potential benefits of diet quality on CV risk factors in diabetic subjects, independent from body weight changes.

Carbohydrates represent the first target of dietary treatment of diabetes. A recent meta-analysis on randomized controlled trials has shown the importance of the amount and types of carbohydrates in the regulation of glucose levels. The results suggest that increasing dietary fiber intake with high-fiber diets or soluble and insoluble fiber supplements may positively influence glucose homeostasis (decreasing fasting blood glucose by $15.32 \mathrm{mg} / \mathrm{dl}$ and $\mathrm{HbAlc}$ by $0.26 \%$ ) in patients with T2D [18•].

Conversely, in a previous meta-analysis [19•] considering the effect of high carbohydrate/low fat diet compared with low carbohydrate/high fat diet, no diet-related effects on $\mathrm{Hb} 1 \mathrm{Ac}$ and blood glucose have been observed. Moreover, the highcarbohydrate diet increases fasting plasma insulin and triglyceride levels by 8 and $13 \%$, respectively, and lowers the HDL cholesterol level by $6 \%$. This meta-analysis excluded all studies in which the high-carbohydrate diet was also high in fiber and, thus, clearly demonstrates that replacement of fat by carbohydrate foods low in fiber may adversely affect insulin resistance and the metabolic profile in patients with T2D. This is at odds with what happens when fat is substituted with carbohydrate foods that are also rich in fiber and/or with low glycemic index, reinforcing the concept that the quality of carbohydrates is more important than the amount from a metabolic point of view.

Among fibers, their different properties seem to be important in relation to glucose and lipid control. Soluble fibers are more effective than insoluble ones in reducing fasting glucose, HbA1c, urinary glucose excretion and serum cholesterol, as recently reviewed by Wolever [20].

\section{Oxidative Stress and Inflammation}

Oxidative stress and inflammation are considered additional pathophysiologycal conditions linked to the development of CVD and T2D.

Epidemiological and clinical evidence shows that diet composition can influence oxidative stress in T2D or high CV-risk subjects, as recently reviewed [21]. In particular, fruit and vegetables reduce oxidative damage and this effect could be triggered by several mechanisms, inducing an improvement of antioxidant defenses and DNA repair enzymes. In addition to 
this, the type of dietary fat influences oxidative balance: monounsaturated fatty acids (MUFA) and saturated fatty acids (SFA) have opposite actions on oxidative stress (beneficial and detrimental, respectively), possibly because of their different chemical properties. Higher amounts of lipoprotein richer in SFA than in unsaturated fatty acids could affect receptor responsiveness in the liver, inducing an increase in low-density lipoprotein levels and making them more prone to oxidation, a condition linked to an increased atherosclerotic plaques formation.

Moving to inflammation, weight-loss-mediated improvement of C-reactive protein (CRP) has been observed [22], whereas trials focusing on quality of diet have provided inconsistent results. A high carbohydrate/high-fiber/low-glycemic index or a high-MUFA diet have shown similar effects on fasting CRP concentrations, whereas a small but significant decrease in postprandial CRP levels has been observed with the high-MUFA diet [23].

The source of fiber could have a critical role in the influence on inflammatory status. In fact, in our experience, a diet rich in whole wheat-fiber products has no effect on inflammation markers [24], whereas de Mello and colleagues [25] have shown that whole rye bread induces a significant reduction in CRP levels. This difference could be also due to other compounds contained in rye products besides fiber.

\section{Liver Fat}

It is well known that T2D is independently associated with nonalcoholic fatty liver disease (NAFLD) [26], a condition that has been recognized as a possible emerging $\mathrm{CV}$ risk factor. Moreover, excessive liver fat content is associated with a reduction of peripheral and hepatic insulin sensitivity that can aggravate metabolic abnormalities in diabetic subjects.

As demonstrated by the Look AHEAD trial, weight loss ( $8 \%$ of body weight) can significantly reduce liver fat in type 2 diabetic subjects after 12 months [27].

However, the quality of the diet can also significantly affect liver fat independent of weight loss; in fact, it has been shown that a MUFA-rich diet is able to reduce liver fat by almost $30 \%$ in type 2 diabetic subjects, without any change in body weight $[28 \bullet \bullet$.

\section{Diet and Cardiovascular Morbidity/Mortality}

The inverse relationship between consumption of a healthy diet (Mediterranean diet, Dietary Approach to Stop Hypertension [DASH] diet, Prudent diet, whose quite similar characteristics are shown in Table 1) and CVD has been found in many large prospective studies in non-diabetic populations [29-31].
Now, some data are available also for diabetic patients (Table 2).

First of all, a greater adherence to a healthy diet, characterized by high consumption of vegetables, fruit, whole grains, nuts and a higher intake of fish relative to meat, poultry and eggs, is associated with a significant reduction of recurrent $\mathrm{CV}$ events $(-20 \%)$ in a large cohort of patients with previous CVD and/or diabetes. These data show that a healthy diet may be important not only in primary prevention, but also in secondary prevention or in high CV-risk individuals, such as diabetic patients. Moreover, the beneficial effects are in addition to those of pharmacological therapy generally used in secondary prevention $[32 \bullet \bullet$.

In a cross-sectional study in a cohort of elderly Japanese diabetics, no significant reduction of diet-related fatal events was observed, even if lower mortality rate was detected in those following a vegetables and fish-rich diet. Furthermore, the survival analyses showed a tendency for worse prognosis in high sugar and fat consumers [33].

The association between diet and mortality in type 1 diabetic subjects has been poorly investigated.

EURODIAB is the first European prospective study considering this task; the results have shown that, in a cohort of almost 2,000 subjects, a $5 \mathrm{~g}$-increase of fiber intake, especially soluble fiber, within the range commonly consumed in patients with type 1 diabetes (11.3-28.3 g/day) is associated with lower CVD mortality (-16\%) and all-cause mortality (-28\%) [34••], confirming the importance of dietary fibers in diabetes management, also concerning type 1 diabetes.

Of course, the association between diet and CV risk in epidemiological studies generates hypotheses, but does not provide a casual relationship.

To answer this question, intervention studies are needed. Very recent evidence from two trials is available on this topic.

First of all, the Look AHEAD [10••] trial has shown that an intensive lifestyle modification program focused on weight reduction is able to improve all $\mathrm{CV}$ risk factors, as reported above, whereas it does not reduce the occurence of $\mathrm{CV}$ events and mortality compared to the usual care group in the long term; this finding has induced an early termination of the trial [35••]. The reasons for the lack of difference in CV events may be different: a) a low rate of events that could have reduced the statistical power of the study; b) an improvement in CV risk factors in the usual care group as well; c) a lower use of statins in the intervention group with higher levels of LDLcholesterol; d) a decrease in body weight may be of paramount importance in the reduction of risk for diabetes, but not so relevant in the reduction of CVD [36, 37].

The results obtained in the PREDIMED study [38••] are completely different; this study was stopped earlier on the basis of clear benefits shown by the two experimental diets. In fact, in this study, two Mediterranean diets supplemented with extravirgin olive oil or nuts were able to significantly reduce (almost 
Table 1 Main characteristics of dietary patterns linked to reduction of cardiovascular disease

\begin{tabular}{|c|c|}
\hline Dietary pattern & Main characteristics \\
\hline $\begin{array}{l}\text { Mediterranean } \\
\text { diet }\end{array}$ & $\begin{array}{l}\uparrow \text { vegetables, legumes, fruit, nuts, whole grains, low-glycemic index foods, low-fat cheese or yogurt, fish, poultry, MUFA (from extra- } \\
\text { virgin olive oil) } \\
\downarrow \text { red meats, refined cereals }\end{array}$ \\
\hline DASH diet & $\begin{array}{l}\uparrow \text { vegetables, legumes, nuts, fruit, whole grains, low-fat dairy products } \\
\downarrow \text { sodium, red and processed meat, high fat dairy products, refined cereals, sweetened beverages }\end{array}$ \\
\hline Prudent diet & $\begin{array}{l}\uparrow \text { vegetables, fruit, legumes, whole grains, fish } \\
\downarrow \text { red and processed meat, butter, high fat dairy products, eggs, refined cereals }\end{array}$ \\
\hline
\end{tabular}

DASH: Dietary Approach to Stop Hypertension; MUFA: monounsaturated fatty acids

$30 \%$ for both diets) the incidence of major CV events compared to the control diet in high-risk individuals, including subjects with T2D ( $n=3,614$, almost $50 \%$ of the total population).

Although the early termination of the trial may lead to an overestimation of treatment effects [39], the results suggest that even small changes in diet composition may be really effective, possibly more than weight reduction, in reducing CVD in high-risk individuals such as T2D subjects.

\section{Conclusions}

The evidence from studies published in the last 3 years, as a whole, reinforces the principles on which dietary guidelines for diabetes are based.

The only dietary intervention study with hard endpoints ( $\mathrm{CV}$ events) performed in a population including a relevant number of diabetic patients shows clear and significant benefits of the Mediterranean dietary pattern, independent from body weight change. The other study with hard endpoints, in which the intervention was performed through an intensive lifestyle education program focused on weight reduction, does not show benefits on $\mathrm{CV}$ events. This lack of effect may have different explanations as reported before, but, certainly, does not minimize the importance of diet and physical activity in the treatment of T2D, since these changes are able to reduce body weight, the need and the cost of medications, and the rate of sleep apnea, and to improve quality of life and in some cases, achieve diabetes remission.

Other long-term intervention studies with hard endpoints based not only on weight reduction but also on changes in dietary composition are needed in diabetic patients, even if these studies are more and more complicated to carry out due to the lack of funds.

Table 2 Diet and cardiovascular outcomes in diabetic patients

\begin{tabular}{|c|c|c|c|c|}
\hline \multicolumn{5}{|c|}{ Observational studies } \\
\hline Reference & Participants & Study design & Follow-up & Outcome \\
\hline$[32 \bullet \bullet]$ & $\begin{array}{l}n=31,546 \\
\text { Diabetics/CVD }\end{array}$ & Prospective & 4.8 years & $\downarrow \mathrm{CV}$ events \\
\hline [33] & $\begin{array}{l}n=912 \\
\text { Type } 2 \text { diabetics }\end{array}$ & Cross-sectional & - & $\begin{array}{l}\downarrow \text { mortality rate with } \uparrow \text { vegetables and fish } \\
\text { Tendency for worse prognosis with } \uparrow \text { sugars and } \\
\quad \text { fats }\end{array}$ \\
\hline$[34 \bullet \bullet]$ & $\begin{array}{l}n=2,108 \\
\text { Type } 1 \text { diabetics }\end{array}$ & Prospective & 7.3 years & $\downarrow \mathrm{CV}$ mortality with $\uparrow$ dietary fiber \\
\hline \multicolumn{5}{|c|}{ Intervention studies } \\
\hline Reference & Participants & Study design & Duration & Outcome \\
\hline$[35 \bullet \bullet]$ & $\begin{array}{l}n=5,145 \\
\text { Diabetics }\end{array}$ & $\begin{array}{l}\text { Parallel groups } \\
\text { a) Intensive lifestyle intervention } \\
\text { b) Support and education } \\
\text { program }\end{array}$ & 4 years & No difference in $\mathrm{CV}$ events \\
\hline$[38 \bullet \bullet]$ & $\begin{array}{l}n=7,447 \\
\text { Diabetics or at least one criteria for MS }\end{array}$ & $\begin{array}{l}\text { Parallel groups } \\
\text { a) MedD + EVO } \\
\text { b) MedD + nuts } \\
\text { c) Control }\end{array}$ & 5 years & $\downarrow \mathrm{CV}$ events for both MedD \\
\hline
\end{tabular}

CV: cardiovascular; CVD: cardiovascular disease; EVO: extra-virgin olive oil; MedD: Mediterranean diet; MS: metabolic syndrome $\downarrow$ : reduction; $\uparrow:$ increase 


\section{Compliance with Ethics Guidelines}

Conflict of Interest Rosalba Giacco, Claudia Vetrani, Ettore Griffo, and Angela A. Rivellese declare that they have no conflict of interest.

Human and Animal Rights and Informed Consent This article does not contain any studies with human or animal subjects performed by any of the authors.

\section{References}

Papers of particular interest, published recently, have been highlighted as:

- Of importance

- Of major importance

1. Franz MJ, Powers MA, Leontos C, et al. The evidence for medical nutrition therapy for type 1 and type 2 diabetes in adults. J Am Diet Assoc. 2010;110:1852-89.

2. Lindström J, Peltonen M, Eriksson JG, et al. High-fibre, low-fat diet predicts long-term weight loss and decreased type 2 diabetes risk: the Finnish Diabetes Prevention Study. Diabetologia. 2006;49:912-20.

3. Laakso M. Hyperglycemia and cardiovascular disease in type 2 diabetes. Diabetes. 1999;48:937-42.

4. von Ruesten A, Feller S, Bergmann MM, Boeing H. Diet and risk of chronic diseases: results from the first 8 years of follow-up in the EPIC-Potsdam study. Eur J Clin Nutr. 2013;67:412-9.

5. InterAct Consortium, Romaguera D, Guevara M, et al. Mediterranean diet and type 2 diabetes risk in the European Prospective Investigation into Cancer and Nutrition (EPIC) study: the InterAct project. Diabetes Care. 2011;34:1913-8.

6. Salas-Salvadó J, Bulló M, Babio N, et al. Reduction in the incidence of type 2 diabetes with the Mediterranean diet: results of the PREDIMED-Reus nutrition intervention randomized trial. Diabetes Care. 2011;34:14-9.

7. Lindström J, Peltonen M, Eriksson JG, et al. Improved lifestyle and decreased diabetes risk over 13 years: long-term follow-up of the randomised Finnish Diabetes Prevention Study (DPS). Diabetologia. 2013;56:284-93.

8. Penn L, White M, Lindström J, et al. Importance of weight loss maintenance and risk prediction in the prevention of type 2 diabetes: analysis of European Diabetes Prevention Study RCT. PLoS One. 2013;8:e57143.

9. Coppell KJ, Kataoka M, Williams SM, et al. Nutritional intervention in patients with type 2 diabetes who are hyperglycaemic despite optimised drug treatment-Lifestyle Over and Above Drugs in Diabetes (LOADD) study: randomised controlled trial. BMJ. 2010;341:c3337.

10. • Look AHEAD Research Group, Wing RR. Long-term effects of a lifestyle intervention on weight and cardiovascular risk factors in individuals with type 2 diabetes mellitus: four-year results of the Look AHEAD trial. Arch Intern Med. 2010;170:1566-75. This is the first clinical trial showing that an intensive lifestyle intervention is able to produce sustained weight loss and improvement in cardiovascular risk factors through 4 years of follow-up in patients with type 2 diabetes.

11. Gregg EW, Chen H, Wagenknecht LE, et al. Association of an intensive lifestyle intervention with remission of type 2 diabetes. JAMA. 2012;308:2489-96.

12. •• Andrews RC, Cooper AR, Montgomery AA, et al. Diet or diet plus physical activity versus usual care in patients with newly diagnosed type 2 diabetes: the Early ACTID randomised controlled trial. Lancet. 2011;378:129-39. This trial suggests an easier approach to induce weight loss and improvement of cardiovascular risk factors in the usual clinical practice.

13. - Ajala O, English P, Pinkney J. Systematic review and meta-analysis of different dietary approaches to the management of type 2 diabetes. Am J Clin Nutr. 2013;97:505-16. Although this meta-analyses has some limits, as reported in the text, it offers a good revision of dietary patterns effects on several cardiovascular risk factors.

14. Mann JI, TeMorenga L. Diet and diabetes revisited, yet again. Am J Clin Nutr. 2013;97:453-4.

15. Rivellese AA, Giacco R, Costabile G. Dietary carbohydrates for diabetics. Curr Atheroscler Rep. 2012;14:563-9.

16. Lim EL, Hollingsworth KG, Aribisala BS, et al. Reversal of type 2 diabetes: normalisation of beta cell function in association with decreased pancreas and liver triacylglycerol. Diabetologia. 2011;54: 2506-14.

17. Malandrucco I, Pasqualetti P, Giordani I, et al. Very-low-calorie diet: a quick therapeutic tool to improve $\beta$ cell function in morbidly obese patients with type 2 diabetes. Am J Clin Nutr. 2012;95:609-13.

18. • Post RE, Mainous 3rd AG, King DE, Simpson KN. Dietary fiber for the treatment of type 2 diabetes mellitus: a meta-analysis. J Am Board Fam Med. 2012;25:16-23. This meta-analysis shows that an increase in fiber, naturally contained in the diet or added, reduces the fasting blood glucose level by $15 \mathrm{mg} / \mathrm{dl}$ and the HbAlc level by $0.26 \%$.

19. - Kodama S, Saito K, Tanaka S, et al. Influence of fat and carbohydrate proportions on the metabolic profile in patients with type 2 diabetes: a meta-analysis. Diabetes Care. 2009;32:959-65. This meta-analysis quantifies the effect of high-carbohydrate/low-fat diets as compared with low-carbohydrate/high-fat diets on insulin, triglyceride, and HDL cholesterol levels.

20. Wolever TM. Is glycaemic index (GI) a valid measure of carbohydrate quality? Eur J Clin Nutr. 2013;67:522-31.

21. Vetrani C, Costabile G, Di Marino L, Rivellese AA. Nutrition and oxidative stress: a systematic review of human studies. Int J Food Sci Nutr. 2013;64:312-26.

22. Belalcazar LM, Haffner SM, Lang W, et al. Lifestyle intervention and/or statins for the reduction of C-reactive protein in type 2 diabetes: from the look AHEAD study. Obesity. 2013;21:944-50.

23. Bozzetto L, De Natale C, Di Capua L, et al. The association of hsCRP with fasting and postprandial plasma lipids in patients with type 2 diabetes is disrupted by dietary monounsaturated fatty acids. Acta Diabetol. 2013;50:273-6.

24. Giacco R, Lappi J, Costabile G et al. Effects of rye and whole wheat versus refined cereal foods on metabolic risk factors: a randomised controlled two-centre intervention study. Clin Nutr. 2013. doi:10. 1016/j.clnu.2013.01.016.

25. de Mello VD, Schwab U, Kolehmainen M, et al. A diet high in fatty fish, bilberries and wholegrain products improves markers of endothelial function and inflammation in individuals with impaired glucose metabolism in a randomised controlled trial: the Sysdimet study. Diabetologia. 2011;54:2755-67.

26. Bozzetto L, Prinster A, Mancini M, et al. Liver fat in obesity: role of type 2 diabetes mellitus and adipose tissue distribution. Eur J Clin Investig. 2011;41:39-44.

27. Lazo M, Solga SF, Horska A, et al. Effect of a 12-month intensive lifestyle intervention on hepatic steatosis in adults with type 2 diabetes. Diabetes Care. 2010;33:2156-63.

28. • Bozzetto L, Prinster A, Annuzzi G, et al. Liver fat is reduced by an isoenergetic MUFA diet in a controlled randomized study in type 2 diabetic patients. Diabetes Care. 2012;35:1429-35. A diet enriched in MUFA may be particular useful for the management of hepatic steatosis in people with type 2 diabetes.

29. Sofi F, Abbate R, Gensini GF, Casini A. Accruing evidence on benefits of adherence to the Mediterranean diet on health: an updated systematic review and meta-analysis. Am J Clin Nutr. 2010;92:1189-96.

30. Salehi-Abargouei A, Maghsoudi Z, Shirani F, Azadbakht L. Effects of dietary approaches to stop hypertension (DASH)-style diet on fatal 
or nonfatal cardiovascular diseases-incidence: a systematic review and meta-analysis on observational prospective studies. Nutrition. 2013;29:611-8.

31. Hu FB, Rimm EB, Stampfer MJ, et al. Prospective study of major dietary patterns and risk of coronary heart disease in men. Am J Clin Nutr. 2000;72:912-21.

32. •• Dehghan M, Mente A, Teo KK, et al. Relationship between healthy diet and risk of cardiovascular disease among patients on drug therapies for secondary prevention: a prospective cohort study of 31 546 high-risk individuals from 40 countries. Circulation. 2012;126: 2705-12. This is a large prospective study showing, in a cohort of 31 , 546 subjects, the importance of the healthy diet in secondary prevention or in high cardiovascular-risk individuals, such as diabetic patients.

33. Iimuro $\mathrm{S}$, Yoshimura $\mathrm{Y}$, Umegaki $\mathrm{H}$, et al. Dietary pattern and mortality in Japanese elderly patients with type 2 diabetes mellitus: does a vegetable- and fish-rich diet improve mortality? An explanatory study. Geriatr Gerontol Int. 2012;12 Suppl 1:59-67.

34. •• Schoenaker DA, Toeller M, Chaturvedi N, et al. Dietary saturated fat and fibre and risk of cardiovascular disease and all-cause mortality among type 1 diabetic patients: the EURODIAB Prospective Complications Study. Diabetologia. 2012;55:2132-41. This is the first prospective study, performed on 2,108 European patients with type 1 diabetes, showing that an increase of $5 \mathrm{~g}$ of total dietary fiber per day is associated with a $16 \%$ reduction in cardiovascular disease mortality and a $28 \%$ reduction in all-cause mortality.

35. .• The Look AHEAD Research Group. Cardiovascular Effects of Intensive Lifestyle Intervention in Type 2 Diabetes. N Engl J Med. 2013;369:145-54. This study shows that an intensive lifestyle intervention does not significantly reduce the occurrence of cardiovascular events and mortality compared to the usual care in overweight/obese diabetics, possibly due to several reasons among which the lack of reduction of $L D L$-cholesterol levels is the most relevant. However, other benefits triggered by intensive lifestyle intervention, such as partial and total remission of diabetes, lower need of insulin therapy, improvement of quality of life, etc, are of paramount importance in this type of patients.

36. Gerstein HC. Do lifestyle changes reduce serious outcomes in diabetes? N Engl J Med. 2013;369:189-90.

37. Arterburn DE, O'Connor PJ. A look ahead at the future of diabetes prevention and treatment. JAMA. 2012;308:2517-8.

38. • Estruch R, Ros E, Salas-Salvadó J, et al. Primary prevention of cardiovascular disease with a Mediterranean diet. N Engl J Med. 2013;368:1279-90. This trial clearly shows the beneficial effect of the Mediterranean diet in the reduction of cardiovascular disease mortality (-30\%).

39. Bassler D, Briel M, Montori VM, et al. Stopping randomized trials early for benefit and estimation of treatment effects: systematic review and meta-regression analysis. JAMA. 2010;303:1180-7. 